\title{
Inner products of box splines and their derivatives
}

\author{
Hendrik Speleers
}

Report TW 639, January 2014

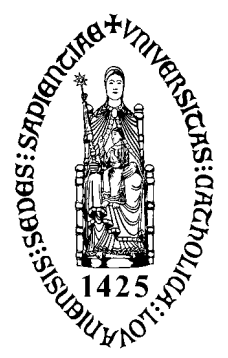

\section{Katholieke Universiteit Leuven} Department of Computer Science 


\title{
Inner products of box splines and their derivatives
}

\author{
Hendrik Speleers
}

Report TW 639, January 2014

Department of Computer Science, KU Leuven

\begin{abstract}
A simple and explicit expression is given for the inner product of (higher order) derivatives of multivariate box splines and their translates. We also show that the energy inner product related to a linear partial differential equation discretized with a set of shifted box splines can be interpreted as a collocation of the problem with a higher order box spline at certain points.
\end{abstract}

Keywords : Box splines, multivariate B-splines, derivatives, inner products, integration.

MSC : Primary : 65D07, Secondary : 41A15, 41A63, 65D30 


\title{
Inner products of box splines and their derivatives
}

\author{
Hendrik Speleers \\ Department of Computer Science, Katholieke Universiteit Leuven \\ Celestijnenlaan 200A, B-3001 Leuven, Belgium
}

\begin{abstract}
A simple and explicit expression is given for the inner product of (higher order) derivatives of multivariate box splines and their translates. We also show that the energy inner product related to a linear partial differential equation discretized with a set of shifted box splines can be interpreted as a collocation of the problem with a higher order box spline at certain points.
\end{abstract}

Keywords: box splines, multivariate B-splines, derivatives, inner products, integration. AMS classification: 41A15, 41A63, 65D07, 65D30

\section{Introduction}

Spline functions are an important tool in many application areas, ranging from geometric modelling to the numerical solution of partial differential equations. For example, splines play a fundamental role in isogeometric analysis [4], a paradigm for numerical simulation which combines finite element analysis with computer-aided design methods. The so-called polyhedral splines are a very general and well established family of multivariate polynomial splines, see e.g. [8]. They are obtained as a volumetric projection or "shadow" of a higher dimensional polytope. Box splines and simplex splines are the most interesting examples, see $[3,14,17]$ and references therein.

Many practical problems require to compute the value of the inner product of two splines. This is usually the case in least-squares approximation methods and in Galerkin finite element methods. Various types of quadrature methods have been developed for this purpose, especially for univariate B-splines and NURBS, see $[1,10,13,18]$. On the other hand, algorithms to compute the exact values of these inner products have also been provided for multivariate simplex splines $[9,15]$ and for multivariate polyhedral splines [5]. Recently, a simple expression has been derived in [7] for the inner product of (higher order) derivatives of univariate cardinal B-splines and their translates. In this note, we generalize the formula for the inner product of derivatives of box splines. To the best of our knowledge, no explicit expressions for such inner products are given in the literature for box splines.

The note is organized as follows. In Section 2 we recall the definition and some main properties of box splines. The main result is derived in Section 3 where we give a simple and explicit expression for the inner product of (higher order) derivatives of box splines and their translates. We also consider a small application in the finite element context: we show that the energy inner product related to a linear partial differential equation discretized with a set of shifted box splines can be interpreted as a collocation of the problem with a higher order box spline at certain points. In Section 4 we conclude with some final remarks. 


\section{Box splines}

In this section we summarize the definition of box splines and some of their main properties. For a more comprehensible treatment, we refer to $[3,17]$ and references therein.

A $d$-variate box-spline $M_{\Xi}(\boldsymbol{x})$ is determined by (possibly repeated) direction vectors $\boldsymbol{v}_{k} \in \mathbb{Z}^{d} \backslash \mathbf{0}$, $k=1, \ldots, n$, collected in a $d \times n$ matrix $\Xi:=\left[\boldsymbol{v}_{1} \cdots \boldsymbol{v}_{n}\right]$. For simplicity, we assume that $n \geq d$ and that $\boldsymbol{v}_{1}, \ldots, \boldsymbol{v}_{d}$ are linearly independent, so they span $\mathbb{R}^{d}$ and $\Xi_{d}:=\left[\boldsymbol{v}_{1} \cdots \boldsymbol{v}_{d}\right]$ is a regular matrix. We denote by $\Xi[0,1)^{n}$ the set of all points in $\mathbb{R}^{d}$ obtained after multiplication of $\Xi$ with any point belonging to the set $[0,1)^{n}$ (in vector notation). Moreover, we use the notation $X \subseteq \Xi$ to indicate that the matrix $X$ is obtained from $\Xi$ by the deletion of some columns. Similarly, $\Xi \cup X:=[\Xi X]$ means the matrix made up of all columns of $\Xi$ and $X$, whereas $\Xi \backslash X$ means the matrix obtained from $\Xi$ by omitting once each column of $X$. Under these assumptions, the box splines can be defined by successive convolutions as follows:

$$
M_{\Xi_{d}}(\boldsymbol{x}):= \begin{cases}1 /\left|\operatorname{det}\left(\Xi_{d}\right)\right|, & \text { if } \boldsymbol{x} \in \Xi_{d}[0,1)^{d} \\ 0, & \text { otherwise }\end{cases}
$$

and

$$
M_{\Xi}(\boldsymbol{x}):=\int_{0}^{1} M_{\Xi \backslash \boldsymbol{v}_{n}}\left(\boldsymbol{x}-t \boldsymbol{v}_{n}\right) \mathrm{d} t, \quad n>d .
$$

Any box spline can be interpreted geometrically as the "shadow" of a parallelepiped (also called box). It is well known that univariate cardinal B-splines and tensor-product B-splines are special box splines.

Theorem 2.1. A box spline $M_{\Xi}(\boldsymbol{x})$ has the following properties:

- its construction does not depend on the ordering of the direction vectors $\boldsymbol{v}_{i}, i=1, \ldots, n$;

- it is positive over the convex set $\Xi[0,1)^{n}$, and its support is given by $\Xi[0,1]^{n}$;

- it is symmetric with respect to the central point $\boldsymbol{m}_{\Xi}:=\left(\boldsymbol{v}_{1}+\cdots+\boldsymbol{v}_{n}\right) / 2$;

- it is $\rho-2$ times continuously differentiable, where $\rho$ is the minimal number of columns that need to be removed from $\Xi$ to obtain a matrix whose columns do not span $\mathbb{R}^{d}$;

- it is a d-variate piecewise polynomial of degree $n-d$.

A box spline is characterizable as a distribution by

$$
\int_{\mathbb{R}^{d}} M_{\Xi}(\boldsymbol{x}) f(\boldsymbol{x}) \mathrm{d} \boldsymbol{x}=\int_{[0,1)^{n}} f(\Xi \boldsymbol{t}) \mathrm{d} \boldsymbol{t},
$$

for all continuous functions $f(\boldsymbol{x})$. Box splines can also be described by means of a recurrence relation. If the box splines $M_{\Xi \backslash \boldsymbol{v}_{k}}, k=1, \ldots, n$, are continuous at $\boldsymbol{x}=\sum_{k=1}^{n} \alpha_{k} \boldsymbol{v}_{k}$, then

$$
M_{\Xi}(\boldsymbol{x})=\frac{1}{n-d} \sum_{k=1}^{n} \alpha_{k} M_{\Xi \backslash \boldsymbol{v}_{k}}(\boldsymbol{x})+\left(1-\alpha_{k}\right) M_{\Xi \backslash \boldsymbol{v}_{k}}\left(\boldsymbol{x}-\boldsymbol{v}_{k}\right)
$$

Stable evaluation algorithms based on this recurrence relation are given in $[2,12]$. An alternative approach is to evaluate after conversion to the Bernstein-Bézier form, see [11].

If the columns of $\Xi \backslash \boldsymbol{v}_{k}$ span $\mathbb{R}^{d}$, then we can compute the directional derivative $D_{\boldsymbol{v}_{k}} M_{\Xi}$ with respect to $\boldsymbol{v}_{k}$ as

$$
D_{\boldsymbol{v}_{k}} M_{\Xi}(\boldsymbol{x})=M_{\Xi \backslash \boldsymbol{v}_{k}}(\boldsymbol{x})-M_{\Xi \backslash \boldsymbol{v}_{k}}\left(\boldsymbol{x}-\boldsymbol{v}_{k}\right) .
$$


There is a well established theory for spaces spanned by integer translates of box splines, namely

$$
\left\{M_{\Xi}(\cdot-i), i \in \mathbb{Z}^{d}\right\}
$$

The elements in the set (2.6) form a partition of unity. Moreover, their linear independence can be characterized in a very elegant way in terms of the matrix $\Xi$. The functions in (2.6) are linearly independent if and only if each regular $d \times d$ submatrix $\bar{\Xi}$ of $\Xi$ is unimodular, i.e.,

$$
\operatorname{det}(\bar{\Xi}) \in\{1,-1\}
$$

We recall that local linear independence is equivalent to (global) linear independence in the case of (2.6). Box splines on three-directional meshes are an important family of linearly independent sets of box splines. A closed formula for box splines on three-directional meshes can be found in [16].

We remark that the central points of the supports of the shifted box splines in (2.6) play the role of Greville points $\boldsymbol{g}_{\Xi, \boldsymbol{i}}$, namely

$$
\boldsymbol{x}=\sum_{\boldsymbol{i} \in \mathbb{Z}^{d}} \boldsymbol{g}_{\Xi, \boldsymbol{i}} M_{\Xi}(\boldsymbol{x}-\boldsymbol{i}), \quad \boldsymbol{g}_{\Xi, \boldsymbol{i}}:=\boldsymbol{m}_{\Xi}+\boldsymbol{i} .
$$

Of course, the relation (2.7) only holds when the linear polynomials belong to the space spanned by (2.6). It is known that the box spline space reproduces all polynomials of degree $\rho-1$, where $\rho$ is defined in Theorem 2.1 .

\section{Inner products}

The next theorem provides an expression for inner products of (shifted) box splines. The result is already known in a slightly different form in the more general context of multivariate polyhedral splines, see [6]. For the sake of completeness, we give a short proof. In the following, we only consider continuous box splines.

Theorem 3.1. Let $M_{\Xi}$ and $M_{\Psi}$ be two box splines as defined in (2.1)-(2.2), where $\Xi$ and $\Psi$ are matrices of size $d \times n$ and $d \times m$ respectively. Then,

$$
\int_{\mathbb{R}^{d}} M_{\Xi}(\boldsymbol{x}) M_{\Psi}(\boldsymbol{x}+\boldsymbol{y}) \mathrm{d} \boldsymbol{x}=M_{\Xi \cup \Psi}\left(2 \boldsymbol{m}_{\Xi}+\boldsymbol{y}\right)=M_{\Xi \cup \Psi}\left(2 \boldsymbol{m}_{\Psi}-\boldsymbol{y}\right) .
$$

Proof. Applying $n$ times the relation (2.2) implies

$$
M_{\Xi \cup \Psi}(\boldsymbol{z})=\int_{[0,1)^{n}} M_{\Psi}(\boldsymbol{z}-\Xi \boldsymbol{t}) \mathrm{d} \boldsymbol{t}
$$

and by setting $f(\boldsymbol{x})=M_{\Psi}(\boldsymbol{z}-\boldsymbol{x})$ in $(2.3)$ we get

$$
M_{\Xi \cup \Psi}(\boldsymbol{z})=\int_{\mathbb{R}^{d}} M_{\Xi}(\boldsymbol{x}) M_{\Psi}(\boldsymbol{z}-\boldsymbol{x}) \mathrm{d} \boldsymbol{x} .
$$

Since $M_{\Psi}$ and $M_{\Xi \cup \Psi}$ are symmetric with respect to their central points $\boldsymbol{m}_{\Psi}$ and $\boldsymbol{m}_{\Xi \cup \Psi}=\boldsymbol{m}_{\Xi}+\boldsymbol{m}_{\Psi}$ respectively, we know

$$
\begin{gathered}
M_{\Psi}(\boldsymbol{z}-\boldsymbol{x})=M_{\Psi}\left(2 \boldsymbol{m}_{\Psi}-\boldsymbol{z}+\boldsymbol{x}\right), \\
M_{\Xi \cup \Psi}\left(2 \boldsymbol{m}_{\Xi}+\boldsymbol{y}\right)=M_{\Xi \cup \Psi}\left(2 \boldsymbol{m}_{\Xi \cup \Psi}-2 \boldsymbol{m}_{\Xi}-\boldsymbol{y}\right)=M_{\Xi \cup \Psi}\left(2 \boldsymbol{m}_{\Psi}-\boldsymbol{y}\right),
\end{gathered}
$$

resulting in (3.1). 
This is an important result to simplify the construction of the mass matrix based on the set of elements in (2.6). In such a case, $\Xi=\Psi$ and $\boldsymbol{y}$ is an integer shift. It follows that the mass matrix is completely known once the box spline $M_{\Xi \cup \Psi}$ has been evaluated at all points in $\mathbb{Z}^{d}$.

We can generalize the previous result by providing an expression for the inner product of (higher order) derivatives of (shifted) box splines. The higher order directional derivative of $M_{\Xi}$ with respect to the directions given as columns in $X$ is denoted by $D_{X} M_{\Xi}$. We first formulate a lemma regarding the (anti-)symmetry of higher order directional derivatives.

Lemma 3.2. Let $M_{\Xi}$ be a box spline as defined in (2.1)-(2.2), where $\Xi$ is a matrix of size $d \times n$, and let $X \subseteq \Xi$ be a matrix of size $d \times r$, so that the columns of $\Xi \backslash X$ span $\mathbb{R}^{d}$. Then,

$$
D_{X} M_{\Xi}\left(\boldsymbol{m}_{\Xi}+\boldsymbol{x}\right)=(-1)^{r} D_{X} M_{\Xi}\left(\boldsymbol{m}_{\Xi}-\boldsymbol{x}\right) .
$$

Proof. It is known that box splines satisfy the symmetry property

$$
M_{\Xi}\left(\boldsymbol{m}_{\Xi}+\boldsymbol{x}\right)=M_{\Xi}\left(\boldsymbol{m}_{\Xi}-\boldsymbol{x}\right) .
$$

The result (3.2) follows from repeated differentiations of this property.

We now arrive at our main result.

Theorem 3.3. Let $M_{\Xi}$ and $M_{\Psi}$ be two box splines as defined in (2.1)-(2.2), where $\Xi$ and $\Psi$ are matrices of size $d \times n$ and $d \times m$ respectively. Let $X \subseteq \Xi$ and $Y \subseteq \Psi$ be two matrices of size $d \times r$ and $d \times s$ respectively, so that the columns of $\Xi \backslash X$ span $\mathbb{R}^{d}$ and the columns of $\Psi \backslash Y$ span $\mathbb{R}^{d}$. Then,

$$
\begin{aligned}
\int_{\mathbb{R}^{d}} D_{X} M_{\Xi}(\boldsymbol{x}) D_{Y} M_{\Psi}(\boldsymbol{x}+\boldsymbol{y}) \mathrm{d} \boldsymbol{x} & =(-1)^{r} D_{X \cup Y} M_{\Xi \cup \Psi}\left(2 \boldsymbol{m}_{\Xi}+\boldsymbol{y}\right) \\
& =(-1)^{s} D_{X \cup Y} M_{\Xi \cup \Psi}\left(2 \boldsymbol{m}_{\Psi}-\boldsymbol{y}\right) .
\end{aligned}
$$

Proof. Because of the (anti-)symmetry of the higher order derivatives of the B-splines given by Lemma 3.2, we have

$$
\begin{aligned}
(-1)^{r} D_{X \cup Y} M_{\Xi \cup \Psi}\left(2 \boldsymbol{m}_{\Xi}+\boldsymbol{y}\right) & =(-1)^{r}(-1)^{r+s} D_{X \cup Y} M_{\Xi \cup \Psi}\left(2 \boldsymbol{m}_{\Xi \cup \Psi}-2 \boldsymbol{m}_{\Xi}-\boldsymbol{y}\right) \\
& =(-1)^{s} D_{X \cup Y} M_{\Xi \cup \Psi}\left(2 \boldsymbol{m}_{\Psi}-\boldsymbol{y}\right) .
\end{aligned}
$$

Hence, we only have to show one of the two equalities in (3.3). This can be proved by induction on the order of derivatives. The base case $(r=0, s=0)$ simply follows from Theorem 3.1. Then, we consider two types of inductive steps: in the inductive step of the first type we increase the order of derivatives of $M_{\Xi}$ by one, i.e., $r-1 \rightarrow r$; while in the inductive step of the second type we increase the order of derivatives of $M_{\Psi}$ by one, i.e., $s-1 \rightarrow s$. Let $\boldsymbol{v} \in X$. By using (2.5) and the induction hypothesis, we have

$$
\begin{aligned}
\int_{\mathbb{R}^{d}} D_{X} & M_{\Xi}(\boldsymbol{x}) D_{Y} M_{\Psi}(\boldsymbol{x}+\boldsymbol{y}) \mathrm{d} \boldsymbol{x} \\
& =\int_{\mathbb{R}^{d}}\left[D_{X \backslash \boldsymbol{v}} M_{\Xi \backslash \boldsymbol{v}}(\boldsymbol{x})-D_{X \backslash \boldsymbol{v}} M_{\Xi \backslash \boldsymbol{v}}(\boldsymbol{x}-\boldsymbol{v})\right] D_{Y} M_{\Psi}(\boldsymbol{x}+\boldsymbol{y}) \mathrm{d} \boldsymbol{x} \\
& =\int_{\mathbb{R}^{d}} D_{X \backslash \boldsymbol{v}} M_{\Xi \backslash \boldsymbol{v}}(\boldsymbol{x}) D_{Y} M_{\Psi}(\boldsymbol{x}+\boldsymbol{y}) \mathrm{d} \boldsymbol{x}-\int_{\mathbb{R}^{d}} D_{X \backslash \boldsymbol{v}} M_{\Xi \backslash \boldsymbol{v}}(\boldsymbol{x}) D_{Y} M_{\Psi}(\boldsymbol{x}+\boldsymbol{y}+\boldsymbol{v}) \mathrm{d} \boldsymbol{x} \\
& =(-1)^{s}\left[D_{X \cup Y \backslash \boldsymbol{v}} M_{\Xi \cup \Psi \backslash \boldsymbol{v}}\left(2 \boldsymbol{m}_{\Psi}-\boldsymbol{y}\right)-D_{X \cup Y \backslash \boldsymbol{v}} M_{\Xi \cup \Psi \backslash \boldsymbol{v}}\left(2 \boldsymbol{m}_{\Psi}-\boldsymbol{y}-\boldsymbol{v}\right)\right] \\
& =(-1)^{s} D_{X \cup Y} M_{\Xi \cup \Psi}\left(2 \boldsymbol{m}_{\Psi}-\boldsymbol{y}\right) .
\end{aligned}
$$


The inductive step of the second type can be proved in a similar way. Let $\boldsymbol{w} \in Y$, then

$$
\begin{aligned}
\int_{\mathbb{R}^{d}} D_{X} & M_{\Xi}(\boldsymbol{x}) D_{Y} M_{\Psi}(\boldsymbol{x}+\boldsymbol{y}) \mathrm{d} \boldsymbol{x} \\
& =\int_{\mathbb{R}^{d}} D_{X} M_{\Xi}(\boldsymbol{x})\left[D_{Y \backslash \boldsymbol{w}} M_{\Psi \backslash \boldsymbol{w}}(\boldsymbol{x}+\boldsymbol{y})-D_{Y \backslash \boldsymbol{w}} M_{\Psi \backslash \boldsymbol{w}}(\boldsymbol{x}+\boldsymbol{y}-\boldsymbol{w})\right] \mathrm{d} \boldsymbol{x} \\
& =\int_{\mathbb{R}^{d}} D_{X} M_{\Xi}(\boldsymbol{x}) D_{Y \backslash \boldsymbol{w}} M_{\Psi \backslash \boldsymbol{w}}(\boldsymbol{x}+\boldsymbol{y}) \mathrm{d} \boldsymbol{x}-\int_{\mathbb{R}^{d}} D_{X} M_{\Xi}(\boldsymbol{x}) D_{Y \backslash \boldsymbol{w}} M_{\Psi \backslash \boldsymbol{w}}(\boldsymbol{x}+\boldsymbol{y}-\boldsymbol{w}) \mathrm{d} \boldsymbol{x} \\
& =(-1)^{r}\left[D_{X \cup Y \backslash \boldsymbol{w}} M_{\Xi \cup \Psi \backslash \boldsymbol{w}}\left(2 \boldsymbol{m}_{\Xi}+\boldsymbol{y}\right)-D_{X \cup Y \backslash \boldsymbol{w}} M_{\Xi \cup \Psi \backslash \boldsymbol{w}}\left(2 \boldsymbol{m}_{\Xi}+\boldsymbol{y}-\boldsymbol{w}\right)\right] \\
& =(-1)^{r} D_{X \cup Y} M_{\Xi \cup \Psi}\left(2 \boldsymbol{m}_{\Xi}+\boldsymbol{y}\right),
\end{aligned}
$$

and the proof is complete.

We can also formulate an equivalent expression for inner products of directional derivatives where the directions do not necessarily belong to $\Xi:=\left[\boldsymbol{v}_{1} \cdots \boldsymbol{v}_{n}\right]$ and $\Psi:=\left[\boldsymbol{w}_{1} \cdots \boldsymbol{w}_{m}\right]$, used in the construction of $M_{\Xi}$ and $M_{\Psi}$. Given any two directions $\boldsymbol{v}, \boldsymbol{w} \in \mathbb{R}^{d} \backslash \mathbf{0}$, and the linear combinations

$$
\boldsymbol{v}=\sum_{k=1}^{n} \alpha_{k} \boldsymbol{v}_{k}, \quad \boldsymbol{w}=\sum_{l=1}^{m} \beta_{l} \boldsymbol{w}_{l}
$$

for some sets of $\alpha_{k}$ and $\beta_{l}$. Because the columns of $\Xi$ (and $\Psi$ ) span $\mathbb{R}^{d}$, the above linear combinations always exist, but are not necessarily unique. Let $M_{\Xi} \in C^{1}$ and $M_{\Psi} \in C^{1}$, then we have

$$
\begin{aligned}
\int_{\mathbb{R}^{d}} D_{\boldsymbol{v}} M_{\Xi}(\boldsymbol{x}) D_{\boldsymbol{w}} M_{\Psi}(\boldsymbol{x}+\boldsymbol{y}) \mathrm{d} \boldsymbol{x} & =\int_{\mathbb{R}^{d}}\left(\sum_{k=1}^{n} \alpha_{k} D_{\boldsymbol{v}_{k}} M_{\Xi}(\boldsymbol{x})\right)\left(\sum_{l=1}^{m} \beta_{l} D_{\boldsymbol{w}_{l}} M_{\Psi}(\boldsymbol{x}+\boldsymbol{y})\right) \mathrm{d} \boldsymbol{x} \\
& =\sum_{k=1}^{n} \sum_{l=1}^{m} \alpha_{k} \beta_{l} \int_{\mathbb{R}^{d}} D_{\boldsymbol{v}_{k}} M_{\Xi}(\boldsymbol{x}) D_{\boldsymbol{w}_{l}} M_{\Psi}(\boldsymbol{x}+\boldsymbol{y}) \mathrm{d} \boldsymbol{x} .
\end{aligned}
$$

From Theorem 3.3 it follows that

$$
\begin{aligned}
\int_{\mathbb{R}^{d}} D_{\boldsymbol{v}} M_{\Xi}(\boldsymbol{x}) D_{\boldsymbol{w}} M_{\Psi}(\boldsymbol{x}+\boldsymbol{y}) \mathrm{d} \boldsymbol{x} & =\sum_{k=1}^{n} \sum_{l=1}^{m} \alpha_{k} \beta_{l}(-1) D_{\boldsymbol{v}_{k} \cup \boldsymbol{w}_{l}} M_{\Xi \cup \Psi}\left(2 \boldsymbol{m}_{\Xi}+\boldsymbol{y}\right) \\
& =-D_{\boldsymbol{v} \cup \boldsymbol{w}} M_{\Xi \cup \Psi}\left(2 \boldsymbol{m}_{\Xi}+\boldsymbol{y}\right) .
\end{aligned}
$$

In a completely analogous way, we also obtain that

$$
\int_{\mathbb{R}^{d}} D_{\boldsymbol{v}} M_{\Xi}(\boldsymbol{x}) D_{\boldsymbol{w}} M_{\Psi}(\boldsymbol{x}+\boldsymbol{y}) \mathrm{d} \boldsymbol{x}=-D_{\boldsymbol{v} \cup \boldsymbol{w}} M_{\Xi \cup \Psi}\left(2 \boldsymbol{m}_{\Psi}-\boldsymbol{y}\right) .
$$

Following a similar line of arguments, we can generalize the result in Theorem 3.3 to inner products of higher order directional derivatives of box splines with arbitrary directions.

Corollary 3.4. Let $M_{\Xi}$ and $M_{\Psi}$ be two box splines as defined in (2.1)-(2.2), where $\Xi$ and $\Psi$ are matrices of size $d \times n$ and $d \times m$ respectively. Assuming $M_{\Xi} \in C^{r}$ and $M_{\Psi} \in C^{s}$, let $X$ and $Y$ be two matrices of size $d \times r$ and $d \times s$ respectively. Then,

$$
\begin{aligned}
\int_{\mathbb{R}^{d}} D_{X} M_{\Xi}(\boldsymbol{x}) D_{Y} M_{\Psi}(\boldsymbol{x}+\boldsymbol{y}) \mathrm{d} \boldsymbol{x} & =(-1)^{r} D_{X \cup Y} M_{\Xi \cup \Psi}\left(2 \boldsymbol{m}_{\Xi}+\boldsymbol{y}\right) \\
& =(-1)^{s} D_{X \cup Y} M_{\Xi \cup \Psi}\left(2 \boldsymbol{m}_{\Psi}-\boldsymbol{y}\right) .
\end{aligned}
$$


The above results might simplify the construction of stiffness matrices based on the set of elements in (2.6) when discretizing partial differential equations by the Galerkin method. This will be briefly illustrated by the following second order elliptic differential equation with homogeneous Dirichlet boundary conditions:

$$
\begin{cases}-\Delta u+\boldsymbol{\beta} \cdot \nabla u+\gamma u=f, & \text { in } \Omega, \\ u=0, & \text { on } \partial \Omega,\end{cases}
$$

where $\Omega \subset \mathbb{R}^{d}$ is a domain with Lipschitz boundary, $f \in L_{2}(\Omega), \boldsymbol{\beta} \in \mathbb{R}^{d}$ and $\gamma \geq 0$. It is well known that the weak form of problem (3.5) is formulated as follows: find $u \in \mathcal{V}:=H_{0}^{1}(\Omega)$ such that

$$
a(u, v)=\mathrm{F}(v), \quad \forall v \in \mathcal{V},
$$

where

$$
a(u, v):=\int_{\Omega}(\nabla u \cdot \nabla v+\boldsymbol{\beta} \cdot \nabla u v+\gamma u v) \mathrm{d} \Omega, \quad \mathrm{F}(v):=\int_{\Omega} f v \mathrm{~d} \Omega .
$$

Let us assume that we use a set of linearly independent elements like in (2.6) to discretize the space $\mathcal{V}$. Then, up to some special boundary treatment, the computation of the energy inner products of the interior basis functions can be reduced to a set of generic inner products. We need to compute $a\left(M_{\Xi}, M_{\Xi}(\cdot-\boldsymbol{i})\right)$, assuming both $M_{\Xi}$ and $M_{\Xi}(\cdot-\boldsymbol{i})$ have their supports completely inside $\Omega$. If $M_{\Xi} \in C^{1}$, then Corollary 3.4 implies

$$
a\left(M_{\Xi}, M_{\Xi}(\cdot-\boldsymbol{i})\right)=-\Delta M_{\Xi \cup \Xi}\left(2 \boldsymbol{m}_{\Xi}+\boldsymbol{i}\right)+\boldsymbol{\beta} \cdot \nabla M_{\Xi \cup \Xi}\left(2 \boldsymbol{m}_{\Xi}+\boldsymbol{i}\right)+\gamma M_{\Xi \cup \Xi}\left(2 \boldsymbol{m}_{\Xi}+\boldsymbol{i}\right) .
$$

This means that the energy inner products $a\left(M_{\Xi}, M_{\Xi}(\cdot-\boldsymbol{i})\right)$ can be interpreted as a collocation of the differential operator in (3.5) with the higher order box spline $M_{\Xi \cup \Xi}$ at the points $\left(2 \boldsymbol{m}_{\Xi}+\boldsymbol{i}\right)$. Note that these points are nothing else than the Greville points $\boldsymbol{g}_{\Xi \cup \Xi, i}$ related to the integer shifts of $M_{\Xi \cup \Xi}$, see (2.7). In view of Theorem 3.3 and Corollary 3.4, it is clear that this result also generalizes to higher order linear partial differential equations.

\section{Closure}

We have derived a simple and explicit expression for the inner product of (higher order) derivatives of multivariate box splines and their translates. As a consequence of this expression, the energy inner product related to a linear partial differential equation discretized with a set of shifted box splines can be interpreted as a collocation of the problem with a higher order box spline at certain points.

The recent work [7] analyzes the spectral properties of stiffness matrices arising in the context of B-spline based Galerkin discretizations of elliptic differential equations. Such an analysis is of interest to design effective preconditioners for Krylov-type techniques (like Conjugate Gradient or GMRES) and multigrid strategies for the solution of the corresponding linear systems. By exploiting the well known properties of box splines and also the above interpretation of the energy inner product of box splines, a similar strategy as in [7] could be followed to provide a precise spectral analysis of stiffness matrices based on box splines.

\section{Acknowledgement}

Hendrik Speleers is a Postdoctoral Fellow of the Research Foundation Flanders (Belgium). 


\section{References}

[1] F. Auricchio, F. Calabrò, T.J.R. Hughes, A. Reali, and G. Sangalli. A simple algorithm for obtaining nearly optimal quadrature rules for NURBS-based isogeometric analysis. Comput. Methods in Appl. Mech. Engrg., 249-252:15-27, 2012.

[2] C. de Boor. On the evaluation of box splines. Numer. Algor., 5:5-23, 1993.

[3] C. de Boor, K. Höllig, and S. Riemenschneider. Box Splines. Springer-Verlag, New York, 1993.

[4] J.A. Cottrell, T.J.R. Hughes, and Y. Bazilevs. Isogeometric analysis: toward integration of $C A D$ and FEA. John Wiley \& Sons, 2009.

[5] W.A. Dahmen and C.A. Micchelli. Computation of inner products of multivariate B-splines. Numer. Funct. Anal. Optim., 3:367-375, 1981.

[6] W.A. Dahmen and C.A. Micchelli. Some remarks on multivariate B-splines. In W. Schempp and K. Zeller, editors, Multivariate Approximation Theory II, pages 81-87. Birkhäuser Basel, 1982.

[7] C. Garoni, C. Manni, F. Pelosi, S. Serra-Capizzano, and H. Speleers. On the spectrum of stiffness matrices arising from isogeometric analysis. Numer. Math., to appear.

[8] T.N.T. Goodman. Polyhedral splines. In W. Dahmen, M. Gasca, and C.A. Micchelli, editors, Computation of Curves and Surfaces, pages 347-382. Kluwer Acad. Publ., Dordrecht, 1990.

[9] T.A. Grandine. The evaluation of inner products of multivariate simplex splines. SIAM J. Numer. Anal., 24:882-886, 1987.

[10] T.J.R. Hughes, A. Reali, and G. Sangalli. Efficient quadrature for NURBS-based isogeometric analysis. Comput. Methods in Appl. Mech. Engrg., 199:321-331, 2010.

[11] M. Kim. and J. Peters. Fast and stable evaluation of box-splines via the BB-form. Numer. Algor., 50:381-399, 2009.

[12] L. Kobbelt. Stable evaluation of box-splines. Numer. Algor., 14:377-382, 1997.

[13] P. Moore and D. Molloy. Efficient energy evaluations for active B-spline/NURBS surfaces. Comput. Aided Design, 47:12-31, 2014.

[14] M. Neamtu. What is the natural generalization of univariate splines to higher dimensions? In T. Lyche and L.L. Schumaker, editors, Mathematical Methods for Curves and Surfaces, pages 355-392. Vanderbilt University Press, Nashville, 2001.

[15] M. Neamtu and C.R. Traas. On computational aspects of simplicial splines. Constr. Approx., 7:209-220, 1991.

[16] E. Neuman. A new formula for box splines on three-directional meshes. Math. Comp., 62:227$229,1994$.

[17] H. Prautzsch and W. Boehm. Box splines. In G. Farin, J. Hoschek, and M.S. Kim, editors, Handbook of Computer Aided Geometric Design. 2002.

[18] A.H. Vermeulen, R.H. Bartels, and G.R. Heppler. Integrating products of B-splines. SIAM J. Sci. Stat. Comput., 13:1025-1038, 1992. 